\title{
Pieles Responsivas en Arquitectura y Sistemas de Integración Info-Ambiental a través de Tecnología Arduino
}

\author{
Responsive Skins in Architecture and Info-Environmental Systems through Arduino Technology
}

\author{
Paulo Pereyra Bonifacio \\ Universidad de la República, Uruguay \\ paulopereyra@farq.edu.uy \\ Fernando García Amen \\ Universidad de la República, Uruguay \\ efe@farq.edu.uy
}

\begin{abstract}
This paper presents a case study consisting in proposing and building a responsive skin that reacts to different environmental impulses using Arduino technology. Thus, we aim to obtain an intelligent, integrated and economic achievement, through digital manufacturing processes and low cost technologies integrated in an architectural project.
\end{abstract}

Keywords: Responsive skins; Info-environmental integration; Digital manufacturing; Constructive processes.

\section{Introducción}

Resulta innegable la importancia de los factores bióticos en la conformación del hábitat diseñado por el hombre. Este bagaje informacional, que a priori se compone de todas las determinantes cualitativas y cuantitativas del entorno, debe traducirse en la naturaleza del objeto diseñado, de manera de lograr la necesaria integración informacional entre el objeto y el entorno en el que se posiciona.

Asimismo, los nuevos materiales y las técnicas de fabricación digital han contribuido a la implementación de diseños más orgánicos, y por ende más capaces de integrar la información ambiental en su propia composición.

En este trabajo, se propone el estudio y la construcción de una piel responsiva capaz de reaccionar ante distintos impulsos del entorno mediante la utilización de la tecnología Arduino.

\section{Marco Teórico}

A través de esta realización prototípica, se integrarán las variables del entorno al comportamiento de una pieza diseñada, que conformará un objeto inteligente, capaz de reaccionar ante los estímulos perceptibles del entorno en el que está inmersa.

El prototipo a construir contará con las siguientes características:

Será modelado digitalmente para su estudio en fase previa mediante la utilización de software Rhinoceros, con posterior parametrizado mediante Grasshopper de modo de optimizar el proceso de fabricación digital, y se evaluará su comportamiento material. De ese modo se Integran las tres instancias de la fabricación digital: ideación, producción, evaluación (cad/cam/cae) al inicio del proceso de diseño.

Contará en su diseño con sensores y demás insumos electrónicos y/o mecánicos, capaces de lograr la ingeniería necesaria para su funcionamiento.

Estará enteramente programado mediante tecnología Arduino, con lo cual se generará un artefacto que estará apto para dar respuesta ante estímulos visuales y/o auditivos a través de los sensores de luz y sonido, adoptando así un comportamiento adaptable a distintas situaciones de estimulación.

\section{Aspectos Metodológicos}

\section{Condiciones de partida}

Se parte de un diseño celular, modular y multiplicable, que puede ser replicado omnidireccionalmente en un solo plano, el cual conforma la "piel" a construir.

Este diseño celular está conformado por un artefacto hexagonal que define una partición radial que la descompone en 6 placas triangulares y que posibilita que cada una de ellas realice un movimiento integrado, armónico y responsivo.

Se pone énfasis en el estudio de la unidad celular, su definición formal, funcionamiento, y caracterización espacial. Asimismo, se contemplarán alternativas espaciales de implementación, en el entorno proyectual arquitectónico en el que se ubicará la piel responsiva. 


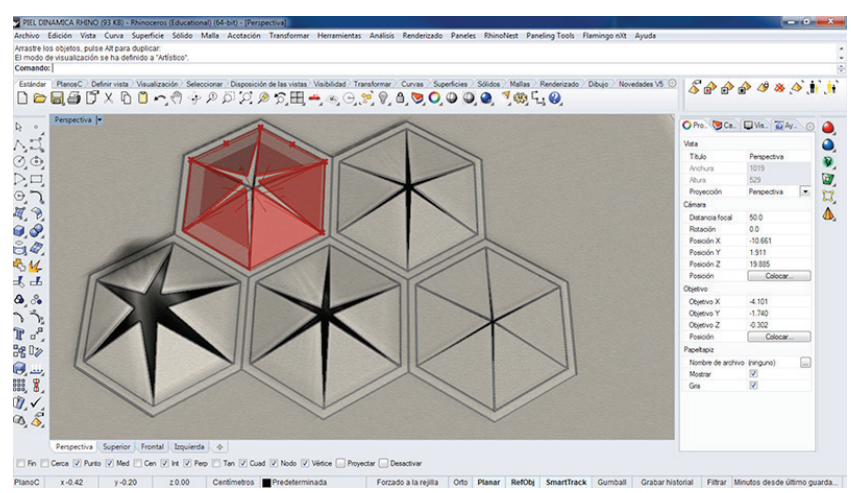

Figura 1: Modelo en Rhinoceros.

\section{Objetivos}

Se propone implementar la solución adoptada en una construcción real, a través de la creación de una piel separatriz entre espacios, dentro del Departamento de Informática de la Facultad de Arquitectura de la UdelaR.

Esta solución cumplirá el doble objetivo de recrear el modelo estudiado en una solución concreta, y a la vez solucionar una necesidad concreta de crear un cerramiento arquitectónico entre dos espacios.

\section{Funcionamiento}

El movimiento de la unidad celular está posibilitada por medio de dos agentes, uno externo y otro interno.

El agente externo posee las siguientes características:

- emite señales analógicas; y

- es variable en el tiempo y en el espacio.

El agente interno, está integrado por un sistema compuesto, conformado por:

- Placas triangulares rebatibles según el eje definido por su encuentro con un bastidor perimetral de forma hexagonal (con un lado tangente a cada cara), diseñado de tal forma que pueda agruparse según plano vertical en nuestro caso de estudio, o eventualmente horizontal en otras posibles aplicaciones.

- Un eje central vinculado a un pivot en forma de racimo.

- El eje central o pistón se encarga de la transmisión del

- movimiento, transformado previamente mediante un mecanismo de engranaje en movimiento axial. El pivot,

- por su parte, se encarga de la distribución de ese

- movimiento axial a las 6 placas triangulares para que las mismas puedan realizar la rotación responsiva.

Las unidades celulares hexagonales poseen, asimismo, los orificios necesarios para ser unidas entre sí con sus pares. Dicha union se realiza mediante tornillos autorroscantes y facilita el armado general de la piel, en formas que se adaptan al espacio que se requiere acondicionar o bien conformar a través de ella. Corresponde acotar, que esta condición contribuye al comportamiento general de la piel, en tanto que unidad arquitectónica construida.

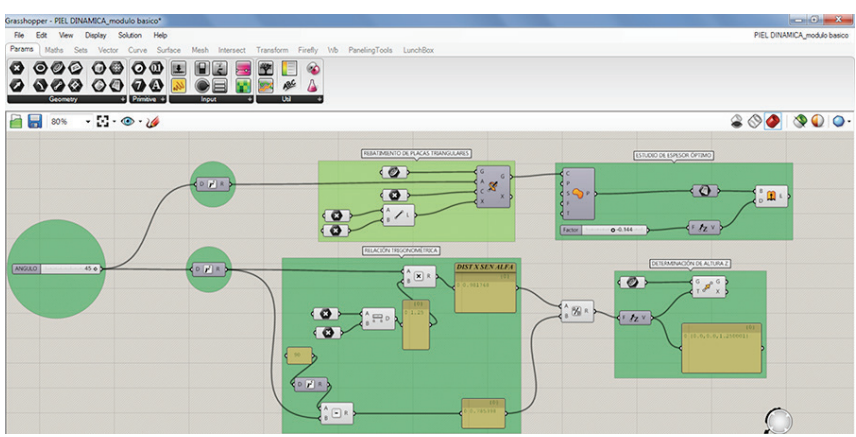

Figura 2: Esquema de funcionamiento en Grasshopper.

\section{Parametrización}

El concepto fundamental que guía la acción es la construcción de un organismo unicelular multiplicable, logrado a través de la concreción de un dispositivo geométrico. Esta construcció, al tiempo que reacciona con un estímulo exterior, mantiene las relaciones internas entre sus componentes siguiendo una estudiada serie de leyes matemáticas.

Dadas entonces estas características que definen geométrica y matemáticamente el dispositivo responsivo, se concluye en la implementación de un diseño paramétrico. Esto se produce como consecuencia de la abstracción de la idea/concepto en que se funda el diseño, ya que permite relacionar procesos evaluando sus resultados en tiempo real y, en caso de ser necesario, modificar los parámetros en los que están contenidas las relaciones establecidas entre el todo y sus partes.

El hecho de utilizar una célula como unidad básica de estudio y el empleo de un patrón geométrico que posibilite su reproducción responde a uno de los objetivos centrales de la investigación

\section{Definición paramétrica}

En el gráfico 01 se aprecia la implementación de un script en donde intervienen como parámetros los siguientes elementos:

- Superficies triangulares;

- Altura del pivot de repartición;

- Ejes definidos por los encuentros entre cada una de las placas y el bastidor;

- Espesores óptimos de placas;

- Ángulo de rebatimiento de las placas, definidas en el dominio de 0 a 45";

- Relación trigonométrica entre ángulo de giro, altura z de pivot y distancia de placas (esta relación surge de despejar, según teorema de senos, el parámetro altura z); 
- Altura $z /$ seno alfa= distancia de placa/seno beta=hipotenusa/seno delta

Por lo tanto: la altura $\mathrm{z}=$ distancia de placa * seno alfa/seno beta. Ver gráfico 02.

\section{Modelo de comportamiento}

El estudio de comportamiento de la célula construida fue efectuado satisfactoriamente aplicando la tecnología Grasshopper; ya que, mediante la implementación del script trigonométrico, fue posible investigar el movimiento del brazo articulador y visualizar la figura de arco capaz. Esa condición cinética condiciona fuertemente el diseño de las placas en cuanto su espesor ya que éste debe "amortiguar" la desviación circular del arco capaz así como canalizar la transmisión del movimiento del pivot. Por otra parte, y mediante la estructuración de un cluster conformado por las células cinéticas básicas en Grasshopper se introduce un "atractor" que posibilita traducir físicamente aquella doble condición establecida al agente exterior. Es decir, un emisor de señales analógicas y la condición de ser variable en el tiempo/espacio.
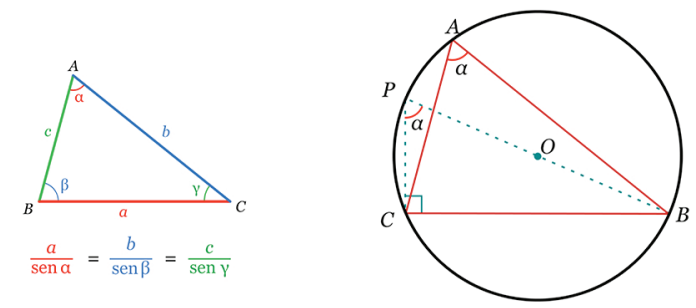

Teorema del seno

Si en un triángulo $\mathrm{ABC}$, las medidas de los lados opuestos a los ángulos $\mathrm{A}, \mathrm{B}$ y $\mathrm{C}$ son respectivamente $\mathrm{a}$,

Ifrac $\{a\}\{\sin \backslash, A\}=\mid$ frac $\{b\}\{\sin \backslash, B\}=$ lfrac $\{\mathcal{C}\}\{\sin ,, C\}$

Figura 3: Teorema del coseno, como fundamento de la apertura.

\section{Emisión de señales analógicas}

Se tradujeron dichas señales mediante dos tecnologías:

- Arduino: hardware microprocesador que, mediante programación de componentes electrónicos permite generar entornos responsivos. En este caso se decodificó la señal de una LDR de modo que la mayor o menor intensidad de luz permitiera una respuesta eficaz de la célula.

- Firefly: un plug-in de Grasshopper que posibilita programar e interactuar con dispositivos periféricos. En particular se investigaron las posibilidades del módulo Skeleton que permite, mediante el dispositivo Kinect, transformar los movimientos corporales en señales digitales y activar el artefacto responsivo.

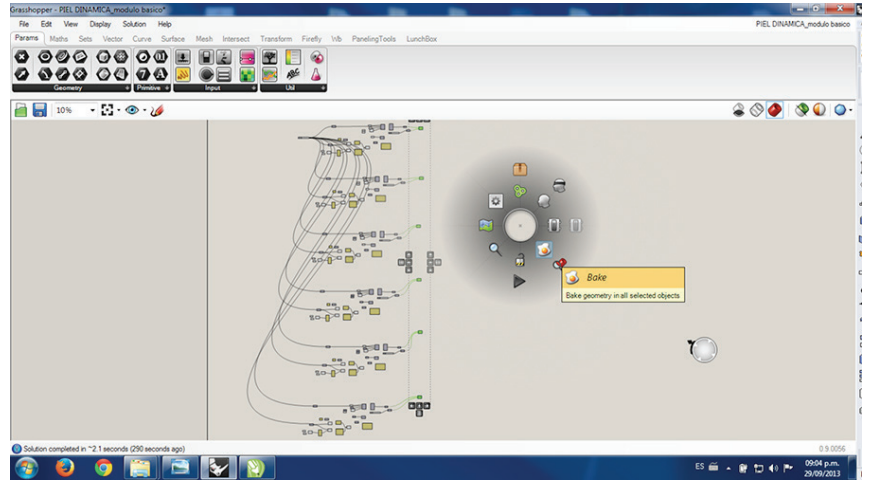

Figura 4: Programación en Grasshopper.

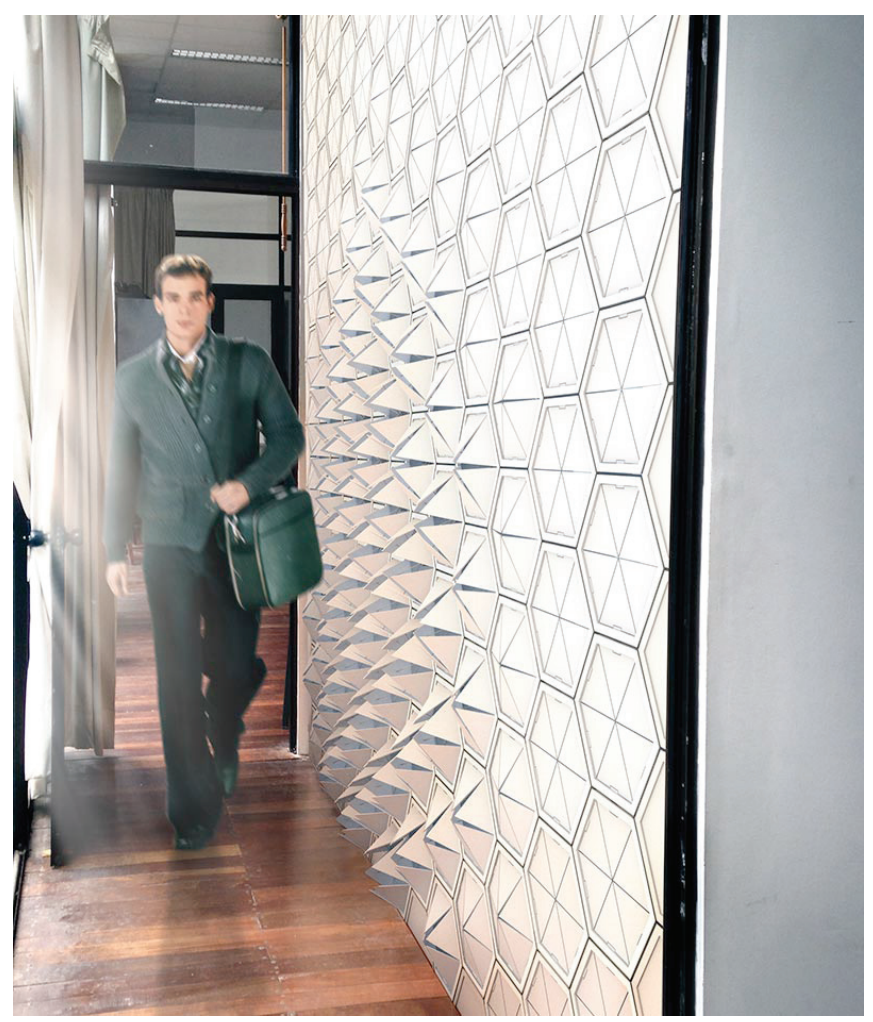

Figura 5: Piel responsiva situada como separatriz entre espacios.

\section{Variabilidad espacio-temporal}

De acuerdo a la distancia del agente externo con respecto al centro de cada célula se establece una condición booleana con respecto al ángulo de rebatimiento de las placas, lo que genera su cierre o apertura.

\section{Fabricación digital}

Las placas triangulares así como el marco bastidor se realizaron con placas de PVC cortadas mediante fresadora CNC utilizando las siguientes técnicas: 
- Perfilado: mediante recortes perimetrales e internos de placas y marcos.

- Rebaje: necesarios para generar rieles de posicionamiento.

Dichas piezas fueron mecanizadas mediante software CAM. Las piezas de transmisión y transferencia, tanto el pistón como el pivot, fueron readaptadas de mecanismos electrónicos reciclados (piezas electrónicas en desuso u obsoletas en su función original), procurando que la solución general logre niveles de satisfacción adecuados mediante la implementación de una estructuración low-cost.

\section{Estímulos}

Las células pueden responder con su movimiento predeterminado (apertura y cierre graduales), ante estimulaciones diversas. En este caso, se han estudiado los estímulos de luz y presencia. En el primer caso, a través de la utilización de sensores de luz trabajando en coordinación con la placa Arduino, para determinar una apertura/cierre directamente proporcional a la cantidad de luz recibida. En el segundo caso, la apertura/cierre del dispositivo es generada por proximidad a través de la colocación de una cámara Kinect. Esta, a través de la lectura del usuario, activa o desactiva los mecanismos de las células más próximas a la figura leída en el dispositivo.

Es pertinente aclarar, que estas dos formas de estimulación de las células son apenas casos de estudio, lo cual no es óbice para explorar diferentes alternativas mediante otro tipo de sensores, ya sea auditivos, táctiles, etc.

\section{Escenarios Posibles}

La posibilidad real de reproducción de las células está garantizada debido a:

- Su bajo coste de producción debido a los materiales de que está compuesta;

- La existencia en el Laboratorio de la tecnología necesaria para construirla (descrita más arriba);

- El bajo coste de las placas Arduino, los sensores y motores empleados, y el reciclaje de chatarra informática del propio Laboratorio.

El único elemento de coste considerable podría ser la cámara Kinect, pero aun teniendo en cuenta su elevado precio, puede justificarse en el total del proyecto, ya que la solución final lograda tiene un coste final igual o menor que la ejecución de un cerramiento tradicional.

Por otra parte, la gama de opciones constructivas que se deriva de este estudio, puede abrir el espectro del diseño espacial y arquitectónico en su sentido más amplio. Las pieles responsivas, independientemente del tipo de estímulo y del tipo de respuesta que brinden, conforman un campo de estudio y abonan el desarrollo de nuevas alternativas a la hora de proyectar. El tiempo dirá si las pieles responsivas se asientan como un elemento tecnológico más dentro de la arquitectura, si se mantendrán como una alternativa low-cost o si por el contrario, se desarrollarán mediante un circuito de producción masivo y de alta gama. De momento, las pequeñas intervenciones arquitectónicas como la propuesta servirán de escaparate para estas tecnologías, y de este modo podrán ser valoradas y eventualmente legitimadas por el destinatario último, es decir, los usuarios.

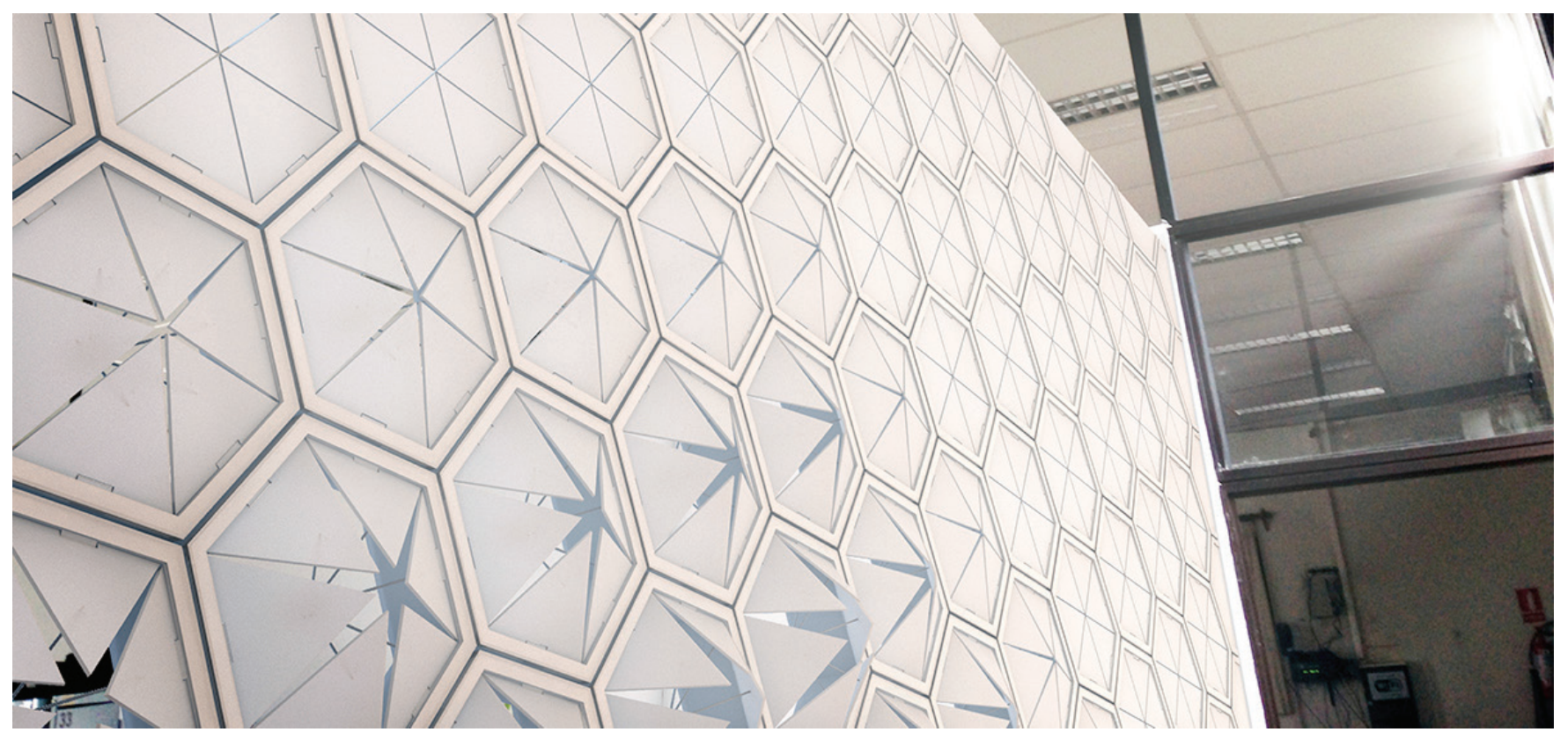

Figura 6: Construcción de células hexagonales con respuesta diversa, dependiendo de la proximidad del usuario. 


\section{Conclusiones}

Esta construcción, allende sus posibilidades técnicas para el diseño en general, aspira a ser un caso de estudio ante las incipientes posibilidades que las nuevas tecnologías constructivas ofrecen a la creación de nuevas arquitecturas y por ende, de nuevos hábitats humanos. En el actual contexto de las Sociedad de la Información, la arquitectura y los procesos constructivos deben integrar simbióticamente las posibilidades tecnológicas y ambientales dentro de parámetros y costes razonables; esto implica, buscar soluciones holísticas para el diseño arquitectónico. Las pieles responsivas son un aporte más en pos de dicha reflexión y un primer paso para alcanzar la integración referida.

\section{Agradecimientos}

Los autores de este artículo desean expresar su agradecimiento a todos aquellos que han colaborado con su desarrollo. Corresponde citar entre estos valiosos aportes a los Bachilleres Andrés Zorrilla de San Martín y Angel Armagno por su participación en el diseño del prototipo; al D.G. Eduardo Cassinelli por sus aportes en mecánica, software libre y diseño de estructuras, el Lic. Camilo Freire y el Bach Nathan Abelar por sus contribuciones y recomendaciones para la programación de la plataforma Arduino; y especialmente a la Bach. Lucía Meirelles, por su incondicional dedicación al proyecto.

\section{Referencias}

MARTINO Gianluca \& MELLIS David (2012). Arduino open source hardware developed by Massimo Banzi, David Cuartielles, and Tom Igoe, http://www.arduino.cc

FRY Ben \& REAS Casey (2012). Processing developed. http://processing.org

RUTTEN David. (2012). Grasshopper developed by David Rutten. http://www.grasshopper3d.com

PAYNE Andy \& JOHNSON Jason. Firefly developed by Andy Payne \& Jason K Johnson. http://www.fireflyexperiments.com/

RUIZ GUTIERREZ, José Manuel (2011). Entornos gráficos para la programación con Arduino. 